\title{
A Stabilized Low Order Finite-Volume Method for the Three-Dimensional Stationary Navier-Stokes Equations
}

\author{
Jian $\mathrm{Li}_{,}{ }^{1,2}$ Xin $\mathrm{Zhao}^{2}{ }^{2}$ Jianhua $\mathrm{Wu}^{1}{ }^{1}$ and Jianhong Yang ${ }^{2}$ \\ ${ }^{1}$ College of Mathematics and Information Science, Shaanxi Normal University, Xi'an 710062, China \\ ${ }^{2}$ Department of Mathematics, Baoji University of Arts and Sciences, Baoji 721007, China \\ Correspondence should be addressed to Jian Li, jiaaanli@gmail.com
}

Received 13 July 2011; Accepted 28 October 2011

Academic Editor: Rafael Martinez-Guerra

Copyright (C) 2012 Jian Li et al. This is an open access article distributed under the Creative Commons Attribution License, which permits unrestricted use, distribution, and reproduction in any medium, provided the original work is properly cited.

This paper proposes and analyzes a stabilized finite-volume method (FVM) for the threedimensional stationary Navier-Stokes equations approximated by the lowest order finite element pairs. The method studies the new stabilized FVM with the relationship between the stabilized FEM (FEM) and the stabilized FVM under the assumption of the uniqueness condition. The results have three prominent features in this paper. Firstly, the error analysis shows that the stabilized FVM provides an approximate solution with the optimal convergence rate of the same order as the usual stabilized FEM solution solving the stationary Navier-Stokes equations. Secondly, superconvergence results on the solutions of the stabilized FEM and stabilized FVM are derived on the $H^{1}$-norm and the $L^{2}$-norm for the velocity and pressure. Thirdly, residual technique is applied to obtain the $L^{2}$-norm error for the velocity without additional regular assumption on the exact solution.

\section{Introduction}

Recently, the development of stable mixed FEMs is a fundamental component in the search for the efficient numerical methods for solving the Navier-Stokes equations governing the flow of an incompressible fluid by using a primitive variable formulation. The object of this work is to analyze the stabilized finite volume method for solving the three-dimensional stationary Navier-Stokes equations.

The importance of ensuring the compatibility of the component approximations of velocity and pressure by satisfying the so-called inf-sup condition is widely understood. The numerous mixed finite elements satisfying the inf-sup condition have been proposed over the years. However, elements not satisfying the inf-sup condition may also work well. So far, the most convenient choice of the finite element space from an implementational point of view 
would be the elements of the low polynomial order in the velocity and the pressure with an identical degree distribution for both the velocity and the pressure.

This paper focuses on the stabilized method called local polynomial pressure projection for the three-dimensional Navier-Stokes equations [1-5]. The proposed method is characterized by the following features. First, the method does not require approximation of derivatives, specification of mesh-dependent parameters, edge-based data structures, and a nonstandard assembly procedure. Second, this method is completely local at the element level.

On the other hand, FVM has become an active area in numerical analysis. The most attractive things are that FVM can keep local conservation and have the advantages of FVM and finite difference methods. The FVM is also termed the control volume method, the covolume method, or the first-order generalized difference method. Nowadays, it is difficult in analyzing FVM to obtain $L^{2}$-norm error estimates because trail functions and test functions are derived from different spaces. Many papers were devoted to its error analysis for secondorder elliptic and parabolic partial differential problems [6-10]. Error estimates of optimal order in the $H^{1}$-norm are the same as those for the linear FEM $[9,11]$. Error estimates of optimal order in the $L^{2}$-norm can be obtained as well $[8,9]$. Moreover, the FVM for generalized Stokes problems was studied by many people [11-13]. They analyzed this method by using a relationship between it and the FEM and obtained its error estimates through those known for the latter method. Also, it still requires $H^{3}$ smoothness assumption of the exact solution to obtain $O\left(h^{2}\right)$ error bound in most previous literatures. However, for the Stokes problems only the finite elements that satisfy the discrete inf-sup condition have been studied.

The work of $[14,15]$ for the two-dimensional stationary Stokes equations is extended in this paper for the three-dimensional stationary Navier-Stokes equations approximated by lowest equal-order finite elements. Following the abstract framework of the relationship between the stabilized FEM and stabilized FVM [14, 15], the stabilized FVM is studied, and the optimal error estimate of the stabilized FVM is obtained for the three-dimensional stationary Navier-Stokes equations relying on the uniqueness condition. As far as known, there still requires much research on FVM results [16] about the velocity in $L^{2}$-norm and superconvergence result between FEM solution and FVM solution of the three-dimensional Navier-Stokes equations.

The remainder of the paper is organized as follows. In Section 2, an abstract functional setting of the three-dimensional Navier-Stokes problem is given with some basic assumptions. In Section 3, the stability of the stabilized FVM is analyzed and provided by Brouwer's fixed-point theorem. In Section 4, the optimal error estimates of the stabilized finite volume approximation for the three-dimensional stationary Navier-Stokes equations are obtained.

\section{FVM Formulation}

Let $\Omega$ be a bounded domain in $R^{3}$, assumed to have a Lipschitz-continuous boundary $\Gamma$ and to satisfy a further condition stated in $(A 1)$ below. The three-dimensional stationary NavierStokes equations are considered as follows:

$$
\begin{gathered}
-v \Delta u+\nabla p+(u \cdot \nabla) u=f, \quad \text { in } \Omega, \\
\operatorname{div} u=0, \quad \text { in } \Omega, \\
\left.u\right|_{\partial \Omega}=0, \quad \text { on } \partial \Omega,
\end{gathered}
$$


where $v>0$ is the viscosity, $u=\left(u_{1}(x), u_{2}(x), u_{3}(x)\right)$ represents the velocity vector, $p=p(x)$ the pressure, and $f=\left(f_{1}(x), f_{2}(x), f_{3}(x)\right)$ the prescribed body force.

In order to introduce a variational formulation, we set [17]

$$
\begin{gathered}
X=\left[H_{0}^{1}(\Omega)\right]^{3}, \quad Y=\left[L^{2}(\Omega)\right]^{3}, \quad M=L_{0}^{2}(\Omega)=\left\{q \in L^{2}(\Omega): \int_{\Omega} q d x=0\right\}, \\
D(A)=\left[H^{2}(\Omega)\right]^{3} \cap X .
\end{gathered}
$$

As mentioned above, a further assumption on $\Omega$ is presented.

(A1) Assume that $\Omega$ is regular so that the unique solution $(v, q) \in(X, M)$ of the steady Stokes problem

$$
-\Delta v+\nabla q=g, \quad \operatorname{div} v=0 \quad \text { in } \Omega,\left.v\right|_{\partial \Omega}=0,
$$

for a prescribed $g \in Y$ exists and satisfies

$$
\|v\|_{2}+\|q\|_{1} \leq c\|g\|_{0}
$$

where $c>0$ is a general constant depending on $\Omega$. Here and after, $\|\cdot\|_{i}$ and $|\cdot|_{i}$ denote the usual norm and seminorm of the Sobolev space $H^{i}(\Omega)$ or $H^{i}(\Omega)^{3}$ for $i=0,1,2$.

We denote by $(\cdot, \cdot)$ the inner product on $L^{2}(\Omega)$ or $Y$. The space $H_{0}^{1}(\Omega)$ and $X$ are equipped with their equivalent scalar product and norm [17]

$$
((u, v))=(\nabla u, \nabla v), \quad\|\nabla u\|_{0}=((u, u))^{1 / 2} .
$$

It is well known [18] that for each $v \in X$ there hold the following inequalities:

$$
\|v\|_{L^{4}} \leq 2^{1 / 2}\|v\|_{0}^{1 / 4}\|\nabla v\|_{0}^{3 / 4}, \quad\|v\|_{0} \leq r\|v\|_{1}
$$

where $\gamma$ is a positive constant depending only on $\Omega$.

The continuous bilinear form $a(\cdot, \cdot)$ on $X \times X$ and $d(\cdot, \cdot)$ on $X \times M$, respectively, are defined by

$$
a(u, v)=((u, v)), \quad \forall u, v \in X, \quad d(v, q)=-(v, \nabla q)=(q, \operatorname{div} v), \quad \forall v \in X, q \in M
$$

Also, the trilinear term is defined by

$$
\begin{aligned}
b(u, v, w) & =((u \cdot \nabla) v, w)+\frac{1}{2}((\operatorname{div} u) v, w) \\
& =\frac{1}{2}((u \cdot \nabla) v, w)-\frac{1}{2}((u \cdot \nabla) w, v), \quad \forall u, v, w \in X
\end{aligned}
$$


and satisfies

$$
b(u, v, w) \leq c_{0}\|\nabla u\|_{0}\|\nabla v\|_{0}\|\nabla w\|_{0}
$$

Then the mixed variational form of $(2.1 \mathrm{a})-(2.1 \mathrm{c})$ is to seek $(u, p) \in(X, M)$ such that

$$
a(u, v)-d(v, p)+d(u, q)+b(u, u, v)=(f, v), \quad \forall(v, q) \in X \times M
$$

The existence and uniqueness results are classical and can be found in [18-20].

We introduce the finite-dimensional subspace $\left(X_{h}, M_{h}\right) \subset(X, M)$, which is characterized by $\tau_{h}$ with mesh scale $h$, a partitioning of $\Omega$ into tetrahedron or hexahedron, assumed to be regular in the usual sense(see [20-22]).

Here, the space $\left(X_{h}, M_{h}\right)$ satisfies the following approximation properties. For each $v \in D(A), p \in H^{1}(\Omega)$, there exist approximations $I_{h} v \in X_{h}$ and $J_{h} q \in M_{h}$ such that

$$
\left\|u-I_{h} u\right\|_{0}+h\left(\left\|\nabla\left(u-I_{h} u\right)\right\|_{0}+\left\|p-J_{h} p\right\|_{0}\right) \leq c h^{2}\left(\|u\|_{2}+\|p\|_{1}\right),
$$

together with the inverse inequality

$$
\left\|\nabla v_{h}\right\|_{0} \leq c_{1} h^{-1}\left\|v_{h}\right\|_{0}, \quad\left\|u_{h}\right\|_{L^{\infty}} \leq c_{2} h^{-1 / 2}\left\|\nabla u_{h}\right\|_{0} .
$$

The stable and accurate finite element approximational solution of (2.10) requires that $\left(X_{h}, M_{h}\right)$ satisfies the discrete inf-sup condition

$$
\sup _{v_{h} \in X_{h}} \frac{d\left(v_{h}, q_{h}\right)}{\left\|\nabla v_{h}\right\|_{0}} \geq \beta\left\|q_{h}\right\|_{0}
$$

where $\beta$ is positive constant independent of $h$.

The main purpose of this paper is to study a stabilized FVM for the stationary 3D Navier-Stokes equations. We follow $[23,24]$ to obtain the dual partition $\widetilde{K}_{h}$. We first choose an arbitrary point $Q$ in the interior of each tetrahedron $\widetilde{K}$ and then connect $Q$ with the barycenters $Q_{i j k}$ of its $2 \mathrm{D}$ faces $\Delta P_{i} P_{j} P_{k}$ by straight lines (see Figure 1). On each face $\Delta P_{i} P_{j} P_{k}$, we connect by straight lines $Q_{i j k}$ with the middle points of the segments $P_{i} P_{j}, P_{j} P_{k}$, and $P_{k} P_{i}$, respectively. Then the contribution of $\widetilde{K}$ to the control volume $\widetilde{K}$ of a vertex $P$ of $\widetilde{K}$ is the volume surrounding $P$ by these straight lines, for example, the contribution from one simplex to the control volume $\tilde{K}$ with the interfaces $\gamma_{12}$ and $\gamma_{13}$.

Then, the dual finite element space can be constructed for the FVM as follows:

$$
\tilde{X}_{h}=\left\{\tilde{v} \in\left[L^{2}(\Omega)\right]^{3}:\left.\widetilde{v}\right|_{\tilde{K}} \in P_{0}(\tilde{K}) \forall \tilde{K} \in \tilde{K}_{h} ;\left.\widetilde{v}\right|_{\partial \tilde{K}}=0\right\}
$$




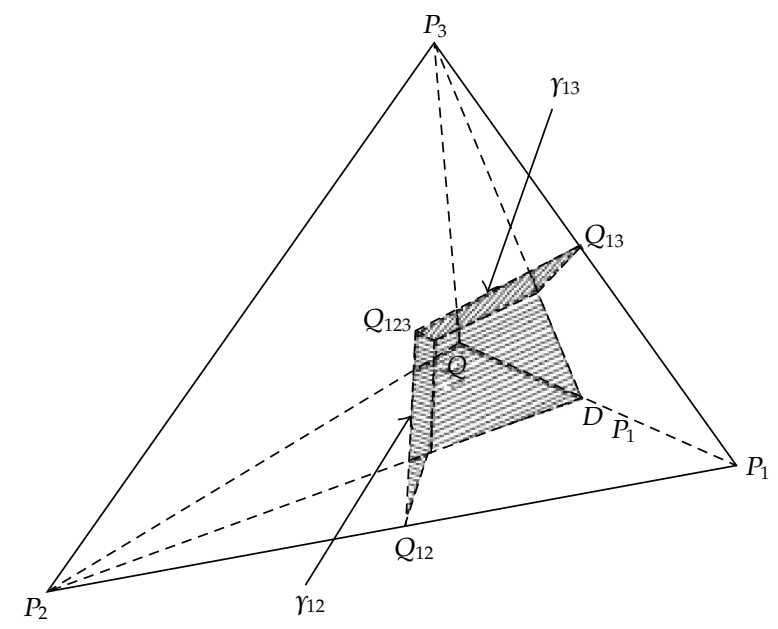

Figure 1: Control volumes in three-dimensional case.

Obviously, the dimensions of $X_{h}$ and $\tilde{X}_{h}$ are the same. Furthermore, there exists an invertible linear mapping $\Gamma_{h}: X_{h} \rightarrow \tilde{X}_{h}$ such that for

$$
v_{h}(x)=\sum_{j=1}^{N} v_{h}\left(P_{j}\right) \phi_{j}(x), \quad x \in \Omega, v_{h} \in X_{h}
$$

with

$$
\Gamma_{h} v_{h}(x)=\sum_{j=1}^{N} v_{h}\left(P_{j}\right) x_{j}(x),
$$

where $\left\{\phi_{j}\right\}$ indicates the basis for the finite element space $X_{h}$, and $\left\{x_{j}\right\}$ denotes the basis for the finite volume space $\tilde{X}_{h}$ that are the characteristic functions associated with the dual partition $\tilde{K}_{h}$ :

$$
x_{j}(x)= \begin{cases}1 & \text { if } x \in \tilde{K}_{j} \in \tilde{K}_{h} \\ 0 & \text { otherwise }\end{cases}
$$

The above idea of connecting the trial and test spaces in the Petrov-Galerkin method through the mapping $\Gamma_{h}$ was first introduced in $[25,26]$ in the context of elliptic problems. Furthermore, the mapping $\Gamma_{h}$ satisfies the following properties [26]. 
Lemma 2.1. Let $K \in K_{h}$. If $v_{h} \in X_{h}$ and $1 \leq r \leq \infty$, then

$$
\begin{gathered}
\int_{K}\left(v_{h}-\Gamma_{h} v_{h}\right) d x=0, \\
\left\|\Gamma_{h} v_{h}\right\|_{0} \leq c_{3}\left\|v_{h}\right\|_{0}, \quad\left\|v_{h}-\Gamma_{h} v_{h}\right\|_{L^{r}(K)} \leq c_{4} h_{K}\left\|\nabla v_{h}\right\|_{L^{r}(K)},
\end{gathered}
$$

where $h_{K}$ is the diameter of the element $K$.

Multiplying (2.1a) by $\Gamma_{h} v_{h} \in \tilde{X}_{h}$ and integrating over the dual elements $\widetilde{K} \in \widetilde{K}_{h},(2.1 \mathrm{~b})$ by $q_{h} \in M_{h}$ and over the primal elements $K \in K_{h}$, and applying Green's formula, we define the following bilinear forms for the FVM:

$$
\begin{gathered}
A\left(u_{h}, \Gamma_{h} v_{h}\right)=-\sum_{j=1}^{N} v_{h}\left(P_{j}\right) \cdot \int_{\partial \tilde{K}_{j}} \frac{\partial u_{h}}{\partial n} d s, \quad u_{h}, v_{h} \in X_{h}, \\
D\left(\Gamma_{h} v_{h}, p_{h}\right)=\sum_{j=1}^{N} v_{h}\left(P_{j}\right) \cdot \int_{\partial \tilde{K}_{j}} p_{h} n d s, \quad p_{h} \in M_{h}, \\
b\left(u_{h}, v_{h}, \Gamma_{h} w_{h}\right)=\left(\left(u_{h} \cdot \nabla\right) v_{h}, \Gamma_{h} w_{h}\right)+\frac{1}{2}\left(\operatorname{div} u_{h} v_{h}, \Gamma_{h} w_{h}\right), \\
\left(f, \Gamma_{h} v_{h}\right)=\sum_{j=1}^{N} v_{h}\left(P_{j}\right) \cdot \int_{\tilde{K}_{j}} f d x, \quad v_{h} \in X_{h},
\end{gathered}
$$

where $n$ is the unit normal outward to $\partial \widetilde{K}_{j}$ and these terms are well posed.

As noted above, this paper forces on a class of unstable velocity-pressure pairs consisting of the lowest equal-order finite elements

$$
\begin{gathered}
X_{h}=\left\{v \in X:\left.v\right|_{K} \in\left[R_{1}(K)\right]^{3}, \forall K \in \tau_{h}\right\}, \\
M_{h}=\left\{q \in M:\left.q\right|_{K} \in R_{i}(K), i=0,1, \forall K \in \tau_{h}\right\},
\end{gathered}
$$

where $R_{i}(K), i=0,1$ represent piecewise constant range and continuous range on set $K, R_{i}$, $i=0,1$ are spaces of polynomials, the maximum degree of which is bounded uniformly with respect to $K \in \tau_{h}$ and $h$. The corresponding stabilized FEM is formulated as follows [3]:

$$
a\left(\bar{u}_{h}, v_{h}\right)-d\left(v_{h}, \bar{p}_{h}\right)+d\left(\bar{u}_{h}, q_{h}\right)+G\left(\bar{p}_{h}, q_{h}\right)+b\left(\bar{u}_{h}, \bar{u}_{h}, v_{h}\right)=\left(f, v_{h}\right), \quad \forall\left(v_{h}, q_{h}\right) \in\left(X_{h}, M_{h}\right) .
$$

Also, the corresponding stabilized FVM is defined for the solution $\left(u_{h}, p_{h}\right) \in\left(X_{h}, M_{h}\right)$ as follows:

$$
\mathcal{C}_{h}\left(\left(u_{h}, p_{h}\right) ;\left(v_{h}, q_{h}\right)\right)+b\left(u_{h}, u_{h}, \Gamma_{h} v_{h}\right)=\left(f, \Gamma_{h} v_{h}\right) \quad \forall\left(v_{h}, q_{h}\right) \in\left(X_{h}, M_{h}\right),
$$


where

$$
\mathcal{C}_{h}\left(\left(u_{h}, p_{h}\right) ;\left(v_{h}, q_{h}\right)\right)=A\left(u_{h}, \Gamma_{h} v_{h}\right)+D\left(\Gamma_{h} v_{h}, p_{h}\right)+d\left(u_{h}, q_{h}\right)+G\left(p_{h}, q_{h}\right) .
$$

Obviously, the bilinear form $G(\cdot, \cdot)$ can be defined by the following symmetry form: [1]

$$
G(p, q)=\left(p-\Pi_{h} p, q-\Pi_{h} q\right)
$$

Note that

$$
\Pi_{h}= \begin{cases}L^{2}(\Omega) \longrightarrow R_{0} & \text { if } i=1 \\ L^{2}(\Omega) \longrightarrow R_{1} & \text { if } i=0 .\end{cases}
$$

Here, the operator $\Pi_{h}$ satisfies the following properties: [1, 4]

$$
\begin{gathered}
\left(p, q_{h}\right)=\left(\Pi_{h} p, q_{h}\right) \quad \forall p \in M, q_{h} \in R_{i}, \\
\left\|\Pi_{h} p\right\|_{0} \leq c_{5}\|p\|_{0} \quad \forall p \in M, \\
\left\|p-\Pi_{h} p\right\|_{L^{p}} \leq c_{6} h\|p\|_{H^{1, p}} \quad \forall p \in H^{1}(\Omega) \cap M .
\end{gathered}
$$

In particular, the $L^{2}$-projection operator $\Pi_{h}$ can be extended to the vector case.

This section concentrates on the study of a relationship between the FEM and FVM for the Stokes equations.

Lemma 2.2. It holds that [11-13]

$$
A\left(u_{h}, \Gamma_{h} v_{h}\right)=a\left(u_{h}, v_{h}\right) \quad \forall u_{h}, v_{h} \in X_{h}
$$

with the following properties:

$$
\begin{gathered}
A\left(u_{h}, \Gamma_{h} v_{h}\right)=A\left(v_{h}, \Gamma_{h} u_{h}\right), \\
\left|A\left(u_{h}, \Gamma_{h} v_{h}\right)\right| \leq c_{7}\left\|\nabla u_{h}\right\|_{0}\left\|\nabla v_{h}\right\|_{0}, \\
\left|A\left(v_{h}, \Gamma_{h} v_{h}\right)\right| \geq v\left\|\nabla v_{h}\right\|_{0}^{2} .
\end{gathered}
$$

Moreover, the bilinear form $D(\cdot, \cdot)$ satisfies [14]

$$
D\left(\Gamma_{h} v_{h}, q_{h}\right)=-d\left(v_{h}, q_{h}\right) \quad \forall\left(v_{h}, q_{h}\right) \in\left(X_{h}, M_{h}\right) .
$$

Based on detailed results on existence, uniqueness, and regularity of the solution for the FVM (2.23), the following result establishes its continuity and weak coercivity. 
Theorem 2.3. It holds that [14]

$$
\begin{array}{r}
\left|\mathcal{C}_{h}\left(\left(u_{h}, p_{h}\right),\left(v_{h}, q_{h}\right)\right)\right| \leq c\left(\left\|u_{h}\right\|_{1}+\left\|p_{h}\right\|_{0}\right)\left(\left\|v_{h}\right\|_{1}+\left\|q_{h}\right\|_{0}\right) \\
\forall\left(u_{h}, p_{h}\right),\left(v_{h}, q_{h}\right) \in\left(X_{h}, M_{h}\right) .
\end{array}
$$

Moreover,

$$
\sup _{\left(v_{h}, q_{h}\right) \in\left(X_{h}, M_{h}\right)} \frac{\left|\mathcal{C}_{h}\left(\left(u_{h}, p_{h}\right),\left(v_{h}, q_{h}\right)\right)\right|}{\left\|v_{h}\right\|_{1}+\left\|q_{h}\right\|_{0}} \geq \beta\left(\left\|u_{h}\right\|_{1}+\left\|p_{h}\right\|_{0}\right) \quad \forall\left(u_{h}, p_{h}\right) \in\left(X_{h}, M_{h}\right),
$$

where $\beta$ is independent of $h$.

\section{Stability}

In this section, we analyze the results of FVM for the three-dimensional stationary NavierStokes equations. Firstly, we are now in a position to show the well-posedness of system (2.23)

$$
h_{0}=\frac{4 c_{2} c_{3} c_{4} r h^{1 / 2}\|f\|_{0}}{v^{2}} .
$$

Theorem 3.1 (stability). For each $h>0$ such that

$$
0<h_{0} \leq \frac{1}{2}
$$

system (2.23) admits a solution $\left(u_{h}, p_{h}\right) \in\left(X_{h}, M_{h}\right)$. Moreover, if the viscosity $v>0$, the body force $f \in Y$, and the mesh size $h>0$ satisfy

$$
0<h_{0} \leq \frac{1}{4}, \quad 1-2 c_{0} c_{5} \gamma v^{-2}\|f\|_{0}>0,
$$

then the solution $\left(u_{h}, p_{h}\right) \in\left(X_{h}, M_{h}\right)$ is unique. Furthermore, it satisfies

$$
\left\|\nabla u_{h}\right\|_{0} \leq \frac{2 c_{3} \gamma}{v}\|f\|_{0^{\prime}} \quad\left\|p_{h}\right\|_{0} \leq \beta^{-1} c_{3} \gamma\|f\|_{0}\left(4 c_{3} c_{8} v^{-2} \gamma\|f\|_{0}+1\right) .
$$

Proof. For fixed $f \in Y$, we introduce the set

$$
B_{M}=\left\{\left(v_{h}, q_{h}\right) \in\left(X_{h}, M_{h}\right):\left\|\nabla u_{h}\right\|_{0} \leq \frac{2 c_{3} \gamma}{v}\|f\|_{0},\left\|p_{h}\right\|_{0} \leq \beta^{-1} c_{3} \gamma\|f\|_{0}\left(4 c_{3} c_{8} v^{-2} \gamma\|f\|_{0}+1\right)\right\}
$$


Then we define the mapping $T_{h}:\left(X_{h}, M_{h}\right) \rightarrow\left(X_{h}, M_{h}\right)$ by [19]

$$
\begin{array}{r}
A\left(P_{h} w_{h}, \Gamma_{h} v_{h}\right)+D\left(\Gamma_{h} v_{h}, p_{h}\right)+d\left(T_{h} w_{h}, q_{h}\right)+G\left(p_{h}, q_{h}\right)+b\left(w_{h}, T_{h} w_{h}, \Gamma_{h} v_{h}\right)=\left(f, \Gamma_{h} v_{h}\right) \\
\forall\left(v_{h}, q_{h}\right) \in\left(X_{h}, M_{h}\right),
\end{array}
$$

where $T_{h}\left(w_{h}, p_{h}\right): \equiv\left(T_{1} w_{h}, T_{2} p_{h}\right)=\left(u_{h}, p_{h}\right)$. We will prove that $T_{h}$ maps $B_{M}$ into $B_{M}$.

First, taking $\left(v_{h}, q_{h}\right)=\left(u_{h}, p_{h}\right) \in\left(X_{h}, M_{h}\right)$ in (3.6), and using (2.12) and (2.19), we see that for for all $\left(v_{h}, q_{h}\right) \in\left(X_{h}, M_{h}\right)$

$$
\begin{aligned}
v\left\|\nabla u_{h}\right\|_{0}^{2} & \leq A\left(u_{h}, \Gamma_{h} u_{h}\right)+G\left(p_{h}, p_{h}\right) \\
& \leq\|f\|_{0}\left\|\Gamma_{h} u_{h}\right\|_{0}+2 c_{2} c_{4} h^{1 / 2}\left\|\nabla w_{h}\right\|_{0}^{2}\left\|\nabla u_{h}\right\|_{0}^{2} \\
& \leq c_{3} \gamma\|f\|_{0}\left\|\nabla u_{h}\right\|_{0}+\frac{4 c_{2} c_{4} c_{3} \gamma h^{1 / 2}\|f\|_{0}}{v}\left\|\nabla u_{h}\right\|_{0}^{2}
\end{aligned}
$$

since

$$
\begin{aligned}
b\left(w_{h}, u_{h}, \Gamma_{h} u_{h}\right) & =b\left(w_{h}, u_{h}, \Gamma_{h} u_{h}-u_{h}\right) \\
& \leq\left(\left\|w_{h}\right\|_{L^{\infty}}\left\|\nabla u_{h}\right\|_{0}+\frac{\sqrt{3}}{2}\left\|u_{h}\right\|_{L^{\infty}}\left\|\nabla w_{h}\right\|_{0}\right)\left\|\Gamma_{h} u_{h}-u_{h}\right\|_{0} \\
& \leq 2 c_{2} c_{4} h^{1 / 2}\left\|\nabla w_{h}\right\|_{0}\left\|\nabla u_{h}\right\|_{0}^{2} .
\end{aligned}
$$

Thus, we have

$$
v\left(1-\frac{4 c_{2} c_{3} c_{4} \gamma h^{1 / 2}\|f\|_{0}}{v^{2}}\right) \leq c_{3} \gamma\|f\|_{0}\left\|\nabla u_{h}\right\|_{0}
$$

which implies

$$
\left\|\nabla u_{h}\right\|_{0} \leq \frac{2 c_{3} \gamma}{v}\|f\|_{0}
$$

Then, using the definition of $b(\cdot ; \cdot, \cdot)$ and $\mathcal{C}_{h}(\cdot, \cdot),(2.19)$, setting $c_{8}=2 \max \left\{2 c_{2} c_{4} h^{1 / 2}, c_{0}\right\}$, and the same approach as above gives that

$$
\begin{aligned}
\frac{\mathcal{C}_{h}\left(\left(u_{h}, p_{h}\right),\left(v_{h}, q_{h}\right)\right)}{\left\|\nabla v_{h}\right\|_{0}+\left\|q_{h}\right\|_{0}} & \geq \beta\left\|p_{h}\right\|_{0}, \\
\left|b\left(w_{h} ; u_{h}, \Gamma_{h} v_{h}\right)\right| & =\left|b\left(w_{h} ; u_{h}, \Gamma_{h} v_{h}-v_{h}\right)+b\left(w_{h} ; u_{h}, v_{h}\right)\right| \\
& \leq c_{8}\left\|\nabla w_{h}\right\|_{0}\left\|\nabla u_{h}\right\|_{0}\left\|\nabla v_{h}\right\|_{0}, \\
\left|\left(f, \Gamma_{h} v_{h}\right)\right| & \leq\|f\|_{0}\left\|\Gamma_{h} v_{h}\right\|_{0} \\
& \leq c_{3} \gamma\|f\|_{0}\left\|\nabla v_{h}\right\|_{0},
\end{aligned}
$$


which, together with (3.10), gives

$$
\left\|p_{h}\right\|_{0} \leq \beta^{-1} c_{3} \gamma\|f\|_{0}\left(4 c_{3} c_{8} \mathcal{v}^{-2} \gamma\|f\|_{0}+1\right) .
$$

Since the mapping $T_{h}$ is well defined, it follows from Brouwer's fixed-point theorem that there exists a solution to system (2.23).

To prove uniqueness, assume that $\left(u_{1}, p_{1}\right)$ and $\left(u_{2}, p_{2}\right)$ are two solutions to $(2.23)$. Then we see that

$$
\mathcal{C}_{h}\left(\left(u_{1}-u_{2}, p_{1}-p_{2}\right),\left(v_{h}, q_{h}\right)\right)+b\left(u_{1}-u_{2} ; u_{1}, \Gamma_{h} v_{h}\right)+b\left(u_{2} ; u_{1}-u_{2}, \Gamma_{h} v_{h}\right)=0
$$

Letting $\left(v_{h}, q_{h}\right)=\left(u_{1}-u_{2}, p_{1}-p_{2}\right)=(e, \eta)$, we obtain

$$
\begin{aligned}
\mathcal{C}_{h}((e, \eta),(e, \eta)) & \geq v\|\nabla e\|_{0}^{2} \\
\left|b\left(e ; u_{1}, \Gamma_{h} e\right)+b\left(u_{2} ; e, \Gamma_{h} e\right)\right| & =\left|b\left(e ; u_{1}, \Gamma_{h} e-e\right)+b\left(e ; u_{1}, e\right)+b\left(u_{2} ; e, \Gamma_{h} e-e\right)\right| \\
& \leq 2 c_{2} c_{4} h^{1 / 2}\left(\left\|\nabla u_{1}\right\|_{0}+\left\|\nabla u_{2}\right\|_{0}\right)\|\nabla e\|_{0}^{2}+c_{0}\left\|\nabla u_{1}\right\|_{0}\|\nabla e\|_{0}^{2} \\
& \leq 2\left(v h_{0}+c_{0} c_{5} \gamma v^{-1}\|f\|_{0}\right)\|\nabla e\|_{0}^{2}
\end{aligned}
$$

which together with (3.3) and (3.13), gives

$$
0 \leq v\left(1-2 c_{0} c_{5} \gamma \mathcal{v}^{-2}\|f\|_{0}\right)\|\nabla e\|_{0}^{2} \leq 0,
$$

which shows that $e=0$ by (3.15); that is, $u_{1}=u_{2}$. Next, applying (3.3) to (3.13) and (2.34) yields that $p_{1}=p_{2}$. Therefore, it follows that (2.23) has a unique solution.

\section{Optimal Error Estimates}

Theorem 4.1 (optimal error and superconvergent results). Assume that $h>0$ satisfies (3.2) and $f \in Y$ and $v>0$ satisfy (3.2). Let $(u, p) \in(X, M)$ and $\left(u_{h}, p_{h}\right) \in\left(X_{h}, M_{h}\right)$ be the solution of (2.10) and (2.23), respectively. Then it holds

$$
\left\|u-u_{h}\right\|_{1}+\left\|p-p_{h}\right\|_{0} \leq \kappa h\left(\|u\|_{2}+\|p\|_{1}+\|f\|_{0}\right) .
$$

Also, if $f \in\left[H^{1}(\Omega)\right]^{3}$, there holds for the solution $\left(\bar{u}_{h}, \bar{p}_{h}\right)$ of $(2.22)$ that

$$
\left\|u_{h}-\bar{u}_{h}\right\|_{1}+\left\|p_{h}-\bar{p}_{h}\right\|_{0} \leq \kappa h^{3 / 2}\left(\|u\|_{2}+\|p\|_{1}+\|f\|_{1}\right) .
$$

Proof. Subtracting (2.10) from (2.23) gives that

$$
\mathcal{C}_{h}\left((e, \eta) ;\left(v_{h}, q_{h}\right)\right)+b\left(e, \bar{u}_{h}, \Gamma_{h} v_{h}\right)+b\left(u_{h}, e, v_{h}\right)+b\left(u_{h}, u_{h}, v_{h}-\Gamma_{h} v_{h}\right)=0
$$


with $(e, \eta)=\left(\bar{u}_{h}-u_{h}, \bar{p}_{h}-p_{h}\right)$. By $\left(v_{h}, q_{h}\right)=(e, \eta)$, it follows that

$$
v\|\nabla e\|_{0}^{2}+G(\eta, \eta)+b\left(e, u_{h}, e\right)+b\left(u_{h}, u_{h}, \Gamma_{h} e-e\right)=\left(f, v_{h}-\Gamma_{h} v_{h}\right)
$$

Using Theorem 3.1, (2.12), (2.23), and (2.25) gives

$$
\begin{aligned}
b\left(e, u_{h}, e\right) & \leq c_{0}\|\nabla u\|_{0}\|\nabla e\|_{0}^{2} \\
& \leq 2 c_{2} c_{4} h^{1 / 2}\left\|\nabla u_{h}\right\|_{0}\|\nabla e\|_{0}^{2} \\
& \leq \frac{4 c_{2} c_{4} c_{5} \gamma h^{1 / 2}\|f\|_{0}}{v^{2}}\|\nabla e\|_{0}^{2} \\
& =v h_{0}\|\nabla e\|_{0}^{2} \\
b\left(u_{h}, u_{h}, \Gamma_{h} e-e\right) & \leq\left|\left(\left(\left(u_{h}-\Pi_{h} u_{h}\right) \cdot \nabla\right) u_{h}+\frac{1}{2} \operatorname{div} u_{h}\left(u_{h}-\Pi_{h} u_{h}\right), e-\Gamma_{h} e\right)\right| \\
& \leq\left\|\nabla u_{h}\right\|_{\infty}\left\|u_{h}-\Pi_{h} u_{h}\right\|_{0}\left\|e-\Gamma_{h} e\right\|_{0} \\
& \leq c h^{3 / 2}\left\|\nabla u_{h}\right\|_{0}\|\nabla e\|_{0}^{2} .
\end{aligned}
$$

Similarly, by Lemma 2.1 and (2.25), we have

$$
\begin{aligned}
\left|\left(f, e-\Gamma_{h} e\right)\right| & =\left|\left(f-\Pi_{h} f, e-\Gamma_{h} e\right)\right| \\
& \leq C h^{i}\|f\|_{i}\left\|e-\Gamma_{h} e\right\|_{0} \\
& \leq c h^{2(i+1)}\|f\|_{i}^{2}+\frac{v_{0}}{4}\|e\|_{1}^{2}, \quad i=0,1 .
\end{aligned}
$$

Combining the above inequalities with (4.3) gives

$$
\|e\|_{1} \leq c\left(h^{3 / 2}+h^{i+1}\right)\|f\|_{i^{\prime}} \quad i=0,1 .
$$

In the same argument, it follows from (2.34) that

$$
\|\eta\|_{0} \leq c\left(h^{3 / 2}+h^{i+1}\right)\|f\|_{i^{\prime}} \quad i=0,1
$$

Noting that [3]

$$
\left\|u-u_{h}\right\|_{1}+\left\|p-p_{h}\right\|_{0} \leq \operatorname{ch}\left(\|u\|_{2}+\|p\|_{1}+\|f\|_{0}\right)
$$

(4.6)-(4.8), and using a triangle inequality completes the proof of Theorem 4.1. 
As noted above, it is still difficult to achieve an optimal error estimate for the velocity in the $L^{2}$-norm for the three-dimensional stationary Navier-Stokes equations. Here, the following dual problem is proposed and analyzed:

$$
a(v, \Phi)+d(v, \Psi)-d(\Phi, q)+b(u ; v, \Phi)+b(v ; u, \Phi)=\left(u-u_{h}, v\right)
$$

Because of convexity of the domain $\Omega$, this problem has a unique solution that satisfies the regularity property [18]

$$
\|\Phi\|_{2}+\|\Psi\|_{1} \leq C\left\|u-u_{h}\right\|_{0} .
$$

Below set $\left(\Phi_{h}, \Psi_{h}\right)=\left(I_{h} \Phi, J_{h} \Psi\right) \in\left(X_{h}, M_{h}\right)$, which satisfies, by (3.2),

$$
\left\|\Phi-\Phi_{h}\right\|_{0}+h\left(\left\|\Phi-\Phi_{h}\right\|_{1}+\left\|\Psi-\Psi_{h}\right\|_{0}\right) \leq C h^{2}\left(\|\Phi\|_{2}+\|\Psi\|_{1}\right)
$$

Theorem 4.2 (optimal $L^{2}$-error for the velocity). Let $(u, p)$ be the solution of $(2.1 \mathrm{a})-(2.1 \mathrm{c})$ and let $\left(u_{h}, p_{h}\right)$ be the solution of (4.3). Then, under the assumptions of Theorem 4.1, it holds

$$
\left\|u-u_{h}\right\|_{0} \leq C h^{2}\left(\|u\|_{2}+\|p\|_{1}+\|f\|_{1}\right) .
$$

Proof. Multiplying (2.1a) and (2.1b) by $\Gamma_{h} \Phi_{h} \in \tilde{X}_{h}$ and $\Psi_{h} \in M_{h}$ and integrating over the dual elements $\tilde{K}$ and the primary elements $K$, respectively, and adding the resulting equations to (2.23) with $\left(v_{h}, q_{h}\right)=\left(\Phi_{h}, \Psi_{h}\right)$, we see that

$$
\begin{aligned}
& A\left(e, \Gamma_{h} \Phi_{h}\right)+D\left(\Gamma_{h} \Phi_{h}, \eta\right)+d\left(e, \Psi_{h}\right)+G\left(\eta, \Psi_{h}\right) \\
& \quad+b\left(e ; u, \Gamma_{h} \Phi_{h}\right)+b\left(u ; e, \Gamma_{h} \Phi_{h}\right)-b\left(e ; e, \Gamma_{h} \Phi_{h}\right)=G\left(p, \Psi_{h}\right)
\end{aligned}
$$

where $(e, \eta)=\left(u-u_{h}, p-p_{h}\right)$. Subtracting (4.14) from (4.10) with $(v, q)=(e, \eta)$ to obtain

$$
\begin{aligned}
\|e\|_{0}^{2}= & a\left(e, \Phi-\Phi_{h}\right)+d\left(e, \Psi-\Psi_{h}\right)-d\left(\Phi-\Phi_{h}, \eta\right)-G\left(\eta, \Psi_{h}\right)+G\left(p, \Psi_{h}\right)+a\left(e, \Phi_{h}\right) \\
& -A\left(e, \Gamma_{h} \Phi_{h}\right)-d\left(\Phi_{h}, \eta\right)-D\left(\Gamma_{h} \Phi_{h}, \eta\right)+b\left(u ; e, \Phi-\Gamma_{h} \Phi_{h}\right)+b\left(e ; u, \Phi-\Gamma_{h} \Phi_{h}\right) \\
& +b\left(e ; e, \Gamma_{h} \Phi_{h}\right) \\
= & a\left(e, \Phi-\Phi_{h}\right)+d\left(e, \Psi-\Psi_{h}\right)-d\left(\Phi-\Phi_{h}, \eta\right)-G\left(\eta, \Psi_{h}\right)+G\left(p, \Psi_{h}\right)+b\left(e ; e, \Gamma_{h} \Phi_{h}\right) \\
& +b\left(u ; e, \Phi-\Gamma_{h} \Phi_{h}\right)+b\left(e ; u, \Phi-\Gamma_{h} \Phi_{h}\right)+\left(f-(u \cdot \nabla) u, \Phi_{h}-\Gamma_{h} \Phi_{h}\right) .
\end{aligned}
$$


Obviously, we deduce from Theorem 3.1, (2.27)-(2.29), (4.11), the inverse inequality (2.12), and the Cauchy inequality that

$$
\begin{aligned}
\left|a\left(e, \Phi-\Phi_{h}\right)+d\left(e, \Psi-\Psi_{h}\right)-d\left(\Phi-\Phi_{h}, \eta\right)\right| & \leq c\left(\|e\|_{1}+\|\eta\|_{0}\right)\left(\left\|\Phi-\Phi_{h}\right\|_{1}+\left\|\Psi-\Psi_{h}\right\|_{0}\right) \\
& \leq c h^{2}\left(\|u\|_{2}+\|p\|_{1}\right)\left(\|\Phi\|_{2}+\|\Psi\|_{1}\right) \\
& \leq c h^{2}\left(\|u\|_{2}+\|p\|_{1}\right)\|e\|_{0}, \\
\left|G\left(\eta, \Psi_{h}\right)-G\left(p, \Psi_{h}\right)\right| & \leq c h\left(\|p-\Pi p\|_{0}+\|\eta\|_{0}\right)\|\Psi\|_{1} \\
& \leq c h^{2}\left(\|u\|_{2}+\|p\|_{1}\right)\|e\|_{0}, \\
\left|\left(f-(u \cdot \nabla) u, \Phi_{h}-\Gamma_{h} \Phi_{h}\right)\right| & =\mid\left(\left[f-\Pi_{h} f\right]\right. \\
& \left.-\left[(u \cdot \nabla) u-\Pi_{h}(u \cdot \nabla) u\right], \Phi_{h}-\Gamma_{h} \Phi_{h}\right) \mid \\
& \leq c h^{2}\left(\|f\|_{1}+\|\nabla[(u \cdot \nabla) u]\|_{0}\right)\left\|\Phi_{h}\right\|_{1} \\
& \leq c h^{2}\left(\|f\|_{1}+\|u\|_{0}^{1 / 2}\|u\|_{2}^{3 / 2}+\|u\|_{1,4}^{2}\right)\|e\|_{0} . \\
\left|b\left(u ; e, \Phi-\Gamma_{h} \Phi_{h}\right)+b\left(e ; u, \Phi-\Gamma_{h} \Phi_{h}\right)\right| & \leq c\|u\|_{2}\|e\|_{1}\left(\left\|\Phi_{h}-\Gamma_{h} \Phi_{h}\right\|_{0}+\left\|\Phi-\Phi_{h}\right\|_{0}\right) \\
& \leq c h^{2}\left(\|u\|_{2}+\|p\|_{1}\right)\|\Phi\|_{1} \\
& \leq c h^{2}\left(\|u\|_{2}+\|p\|_{1}\right)\|e\|_{0}, \\
\left|b\left(e ; e, \Gamma_{h} \Phi_{h}\right)\right| & =\left|b\left(e ; e, \Gamma_{h} \Phi_{h}-\Phi_{h}\right)+b\left(e ; e, \Phi_{h}\right)\right| \\
& \leq c\left(\|e\|_{0,4}\|e\|_{1}\left\|\Gamma_{h} \Phi_{h}-\Phi_{h}\right\|_{0,4}+\|e\|_{1}^{2}\left\|\Phi_{h}\right\|_{1}\right) \\
& \leq c h\|e\|_{0}^{1 / 4}\|e\|_{1}^{7 / 4}\left\|\nabla \Phi_{h}\right\|_{0,4}+c\|e\|_{1}^{2}\left\|\Phi_{h}\right\|_{1} \\
& \leq c h^{2}\left(\|u\|_{2}+\|p\|_{1}\right)\|e\|_{0} .
\end{aligned}
$$

Combining all these inequalities with (4.15) yields (4.13).

In this paper, we have obtained optimal and convergent results of the stabilized mixed finite volume method for the stationary Navier-Stokes equations approximated by the low order finite elements. Furthermore, we could apply the same technique presented to develop and obtain the corresponding results of other (stabilized) mixed finite volume methods in two or three dimensions.

\section{Acknowledgments}

This research was supported in part by the NSF of China (no. 11071193 and 10971124), Program for New Century Excellent Talents in University, Natural Science New Star of Science and Technologies Research Plan in Shaanxi Province of China (no. 2011kjxx12), Research Program of Education Department of Shaanxi Province (no. 11JK0490), the project sponsored by SRF for ROCS, SEM, and Key project of Baoji University of Arts and Science (no. ZK11157). 


\section{References}

[1] P. Bochev, C. R. Dohrmann, and M. D. Gunzburger, "Stabilization of low-order mixed finite elements for the Stokes equations," SIAM Journal on Numerical Analysis, vol. 44, no. 1, pp. 82-101, 2006.

[2] P. Bochev, C. R. Dohrmann, and M. D. Gunzburger, "A computational study of stabilized, low-order $\mathrm{C}^{0}$ finite element approximations of Darcy equations," Computational Mechanics, vol. 38, no. 4, pp. 323-333, 2006.

[3] Y. He and J. Li, "A stabilized finite element method based on local polynomial pressure projection for the stationary Navier-Stokes equations," Applied Numerical Mathematics, vol. 58, no. 10, pp. 1503-1514, 2008.

[4] J. Li and Y. He, "A stabilized finite element method based on two local Gauss integrations for the Stokes equations," Journal of Computational and Applied Mathematics, vol. 214, no. 1, pp. 58-65, 2008.

[5] J. Li, Y. He, and Z. Chen, "A new stabilized finite element method for the transient Navier-Stokes equations," Computer Methods in Applied Mechanics and Engineering, vol. 197, pp. 22-35, 2007.

[6] S. H. Chou and Q. Li, "Error estimates in $\mathrm{L}^{2}, \mathrm{H}^{1}$ and $\mathrm{L}^{\infty}$ and in co-volume methods for elliptic and parabolic problems: a unified approach," Mathematics of Computation, vol. 69, no. 229, pp. 103-120, 2000.

[7] S. H. Chou and P. S. Vassilevski, "A general mixed covolume framework for constructing conservative schemes for elliptic problems," Mathematics of Computation, vol. 68, no. 227, pp. 991-1011, 1999.

[8] Z. Chen, R. Li, and A. Zhou, "A note on the optimal $\mathrm{L}^{2}$-estimate of the finite volume element method," Advances in Computational Mathematics, vol. 16, no. 4, pp. 291-303, 2002.

[9] R. E. Ewing, T. Lin, and Y. Lin, "On the accuracy of the finite volume element method based on piecewise linear polynomials," SIAM Journal on Numerical Analysis, vol. 39, no. 6, pp. 1865-1888, 2002.

[10] R. E. Ewing, R. Lazarov, Y. Lin et al., "Finite volume element approximations of nonlocal reactive flows in porous media," Numerical Methods for Partial Differential Equations, vol. 16, no. 3, pp. 285-311, 2000.

[11] X. Ye, "On the relationship between finite volume and finite element methods applied to the Stokes equations," Numerical Methods for Partial Differential Equations, vol. 17, no. 5, pp. 440-453, 2001.

[12] S. H. Chou and D. Y. Kwak, "Analysis and convergence of a MAC-like scheme for the generalized Stokes problem," Numerical Methods for Partial Differential Equations, vol. 13, no. 2, pp. 147-162, 1997.

[13] S. H. Chou and D. Y. Kwak, "A covolume method based on rotated bilinears for the generalized Stokes problem," SIAM Journal on Numerical Analysis, vol. 35, no. 2, pp. 494-507, 1998.

[14] J. Li and Z. Chen, "A new stabilized finite volume method for the stationary Stokes equations," Advances in Computational Mathematics, vol. 30, no. 2, pp. 141-152, 2009.

[15] J. Li, L. Shen, and Z. Chen, "Convergence and stability of a stabilized finite volume method for the stationary Navier-Stokes equations," BIT Numerical Mathematics, vol. 50, no. 4, pp. 823-842, 2010.

[16] G. He and Y. He, "The finite volume method based on stabilized finite element for the stationary Navier-Stokes problem," Journal of Computational and Applied Mathematics, vol. 205, no. 1, pp. 651-665, 2007.

[17] R. A. Adams, Sobolev Spaces, Academic Press, New York, NY, USA, 1975.

[18] R. Temam, Navier-Stokes Equations, North-Holland, Amsterdam, The Netherlands, 1984.

[19] Y. He, A. Wang, and L. Mei, "Stabilized finite-element method for the stationary Navier-Stokes equations," Journal of Engineering Mathematics, vol. 51, no. 4, pp. 367-380, 2005.

[20] V. Girault and P.-A. Raviart, Finite Element Methods for Navier-Stokes Equations: Theory and Algorithms, Springer, Berlin, Germany, 1986.

[21] P. G. Ciarlet, The Finite Element Method for Elliptic Problems, North-Holland, Amsterdam, The Netherlands, 1978.

[22] Z. Chen, Finite Element Methods and Their Applications, Springer, Heidelberg, Germany, 2005.

[23] C. Carstensen, R. Lazarov, and S. Tomov, "Explicit and averaging a posteriori error estimates for adaptive finite volume methods," SIAM Journal on Numerical Analysis, vol. 42, no. 6, pp. 2496-2521, 2005.

[24] J. Xu and Q. Zou, "Analysis of linear and quadratic simplicial finite volume methods for elliptic equations," Numerische Mathematik, vol. 111, no. 3, pp. 469-492, 2009.

[25] R. Li and P. Zhu, "Generalized difference methods for second order elliptic partial differential equations (I)-triangle grids," Numerical Mathematics-A Journal of Chinese Universities 2, pp. 140-152, 1982.

[26] R. Ewing, R. Lazarov, and Y. Lin, “Finite volume element approximations of nonlocal reactive flows in porous media," Numerical Methods for Partial Differential Equations, vol. 16, no. 3, pp. 285-311, 2000. 


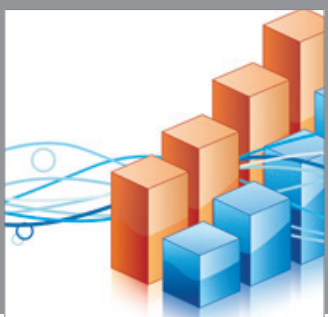

Advances in

Operations Research

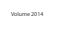

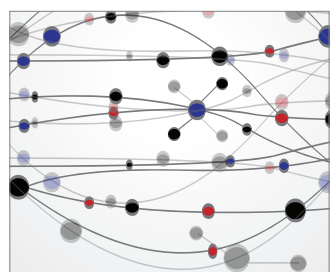

\section{The Scientific} World Journal
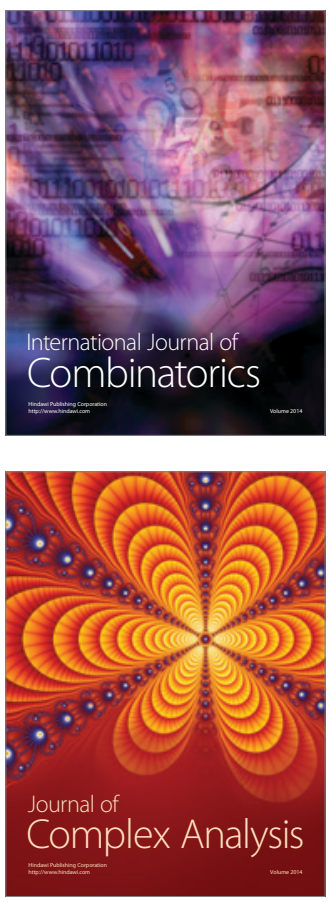

International Journal of

Mathematics and

Mathematical

Sciences
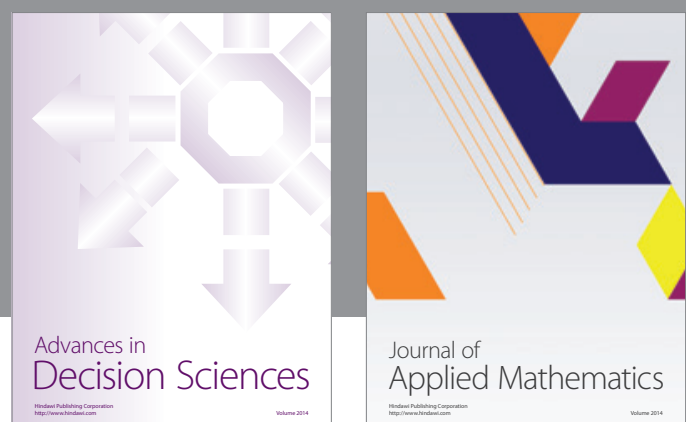

Journal of

Applied Mathematics
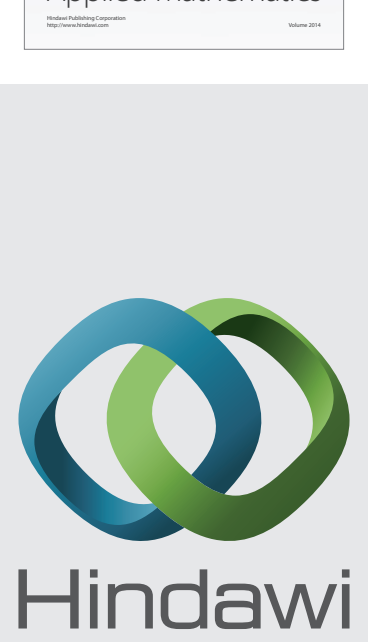

Submit your manuscripts at http://www.hindawi.com
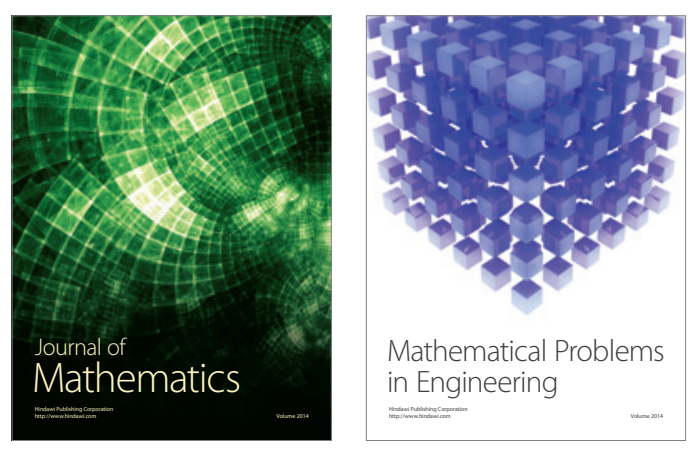

Mathematical Problems in Engineering
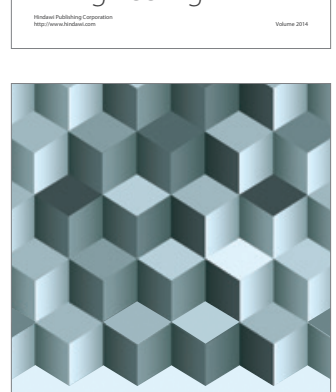

Journal of

Function Spaces
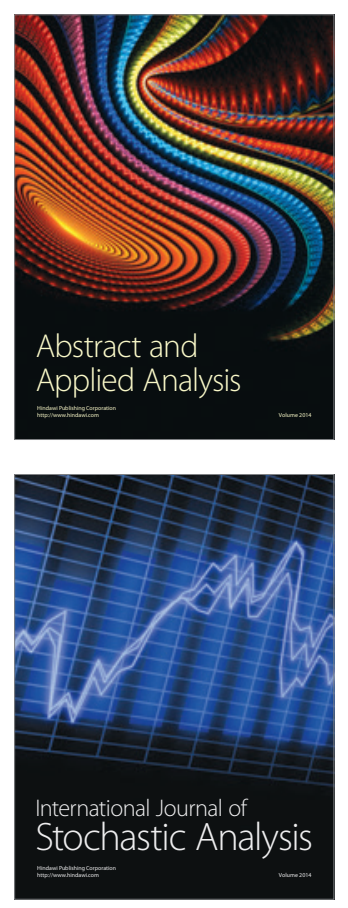

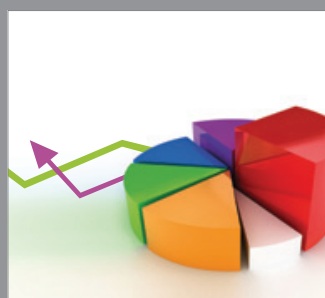

ournal of

Probability and Statistics

Promensencen
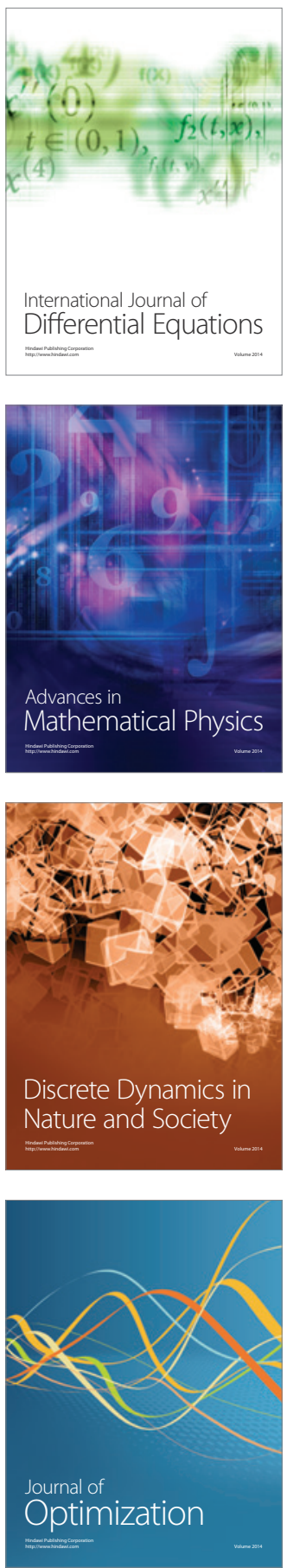